\title{
Memory for truths and falsehoods
}

\author{
MURRAY GLANZER and S. L. EHRENREICH \\ New York University, New York, New York 10003
}

\begin{abstract}
Three experiments were carried out to determine whether recognition memory was more efficient for true statements (on the basis of the subjects' stable body of knowledge) than for false statements. The first two experiments did not show any difference. In a third experiment, the distractors were structured to prevent subjects from responding on the basis of the familiarity of individual component words rather than on the basis of the complete sentences. This experiment showed a clear difference between sentence classes, with true sentences remembered more accurately and faster than false sentences. The data fit, in general, the hypothesis that true sentences are doubly represented in memory. The relation of these findings to the findings on the effects of encoding tasks is also noted.
\end{abstract}

A subject is shown a series of statements. Some he knows are true; some he knows are false. When he is tested later on whether he remembers having seen the statements, which will he remember better, the true or the false? The question is a simple one. It is, however, relevant to other current questions in the area of memory, as will be indicated below.

A standard distinction current in the literature on memory is that between the stable body of knowledge that an individual has and his memory for current, personal events. Included in the former is his knowledge of his language, logic, historical and geographical facts, and laws of nature. Depending on what part of this fund of knowledge is focused on, it is called semantic, generic, categorical, or encyclopedic memory. It will be simply called knowledge here. Contrasted with the individual's knowledge is his memory for current, personal events, referred to as episodic memory (Tulving, 1972).

Recent work on the effect of encoding operations and depth of processing (Craik \& Lockhart, 1972) has been largely concerned with the effect of knowledge on memory, for example, how a subject's knowledge of a word's meaning or its spelling affects his memory for the word. The work has also been concerned with the effect of questions concerning accidental or episodic characteristics of the word's presentation, for example, the typeface used in presentation. The work has established two points: (1) A query that requires the subject to respond with respect to his basic knowledge of a word markedly improves both recognition and

This study was carried out under Grant BNS 76-04923 from the National Science Foundation. Experiments 1 and 2 and the pilot work for Experiment 3 were carried out by Helen Greene. Experiment 3 was carried out by S. L. Ehrenreich. The authors thank Nancy Bowles for her suggestions for that experiment and her comments on the paper. Requests for reprints should be sent to Murray Glanzer, Department of Psychology, New York University, 6 Washington Place, Room 550, New York, New York 10003. recall, and both intentional and incidental memory (Craik \& Tulving, 1975). By contrast, a query with respect to an accidental characteristic produces a relatively weak effect on memory. (2) When the subject makes a positive response to a query about his basic knowledge of a word, he remembers the word better than if he makes a negative response (Craik \& Tulving, 1975; Glanzer \& Koppenaal, 1977; Moscovitch \& Craik, 1976; Schulman, 1971, 1974). This difference does not appear in the case of query about accidental characteristics.

Investigators have discussed the first effect as due to the "depth of processing" induced by the query. They have discussed the second effect as due to the "congruence" of words that produce a positive response with other words in the query.

The mechanism underlying the first effect is, in theory, fairly clear, although attempts to give details of the mechanism have resulted in a rather complicated picture (Craik \& Tulving, 1975). A full review of the problems of the depth-of-processing approach is given by Baddeley (1978).

The mechanism underlying the second effect is less clear. Moscovitch and Craik (1976) tried to determine whether congruence could be reduced to the memorability of different types of questions. Their results do not support the reduction.

There is, however, another way to look at the relation between the subject's knowledge and his episodic memory. This view may be helpful in understanding why knowledge queries are more effective than queries about accidental characteristics and why, within knowledge queries, positive queries are more effective than negative. It may be that processing of information by a subject does two things. It sets up a representation in episodic memory and it also marks the related items or propositions in the subject's body of knowledge, if such items or propositions exist. The last phrase is important. Certain items would have, then, a double representation. Others that do not have relevant proposi- 
tions present in the subject's body of knowledge would have only one representation. For example, a subject who is presented with the word "camel" and the question "Is it an animal?" would have a double representation, one the standard episodic representation, the other the new marking of the proposition "A camel is an animal" in his store of knowledge. A subject presented with the word "camel" and the question "Is it a machine?" would have only one relevant representation since "A camel is not a machine" or any such proposition is not likely to be held in his body of knowledge. In the first case above, with two representations relevant to the recall or recognition of the word "camel," it would be expected that the subject would do better than in a case without such double representation.

The new idea involved here is that of the marking of information in the store of knowledge. The idea is not unreasonable. Indeed, certain data support the idea. For example, repetition of lexical decisions for a word reduces the latency of the decisions for relatively long periods of time (Scarborough, Cortese, \& Scarborough, 1977). Since this long-term reduction affects only words and not nonwords, it is reasonable to assume that the reduction is related to an effect on semantic memory.

One of the implications of this point of view is that statements that are true, with respect to a subject's knowledge (i.e., are represented in it) should be easier to remember than statements that are false. The word "remember" here refers to a standard episodic memory task. This implication will be tested in the studies described below.

\section{EXPERIMENTS 1 and 2}

The first two experiments are almost identical in method and results and will be briefly described together here. The description of the method is relevant as an introduction to Experiment 3 . The results are also useful as an introduction to that experiment. In both, subjects were given a set of statements to study, half true, half false. They read each statement and then were given a standard yes-no recognition test.

\section{Method}

Materials. For both experiments, the study sets contained 96 matched pairs of sentences. Each pair consisted of one true and one false statement on the same general topic, with the pairs covering a range of different types of information: historical, categorical, biological, geographical, occupational, and so on. These are shown in Table 1. Half of the true statements were assigned to one study list, $A$. The other half were assigned to

Table 1

A puck is used in hockey. Football is an outdoor sport.

Moscow is in Russia.

Newton was a scientist.

Books are kept in libraries.
A bow is used in basketball. Poker is a dance. Berlin is in Cuba.

Beethoven was a sculptor. Spices are kept in armories.
Table 2

Proportion of Hits and False Alarms in Experiments 1 and 2

\begin{tabular}{|c|c|c|c|c|c|}
\hline \multirow[b]{2}{*}{$\begin{array}{c}\text { Experi- } \\
\text { ment }\end{array}$} & \multicolumn{2}{|c|}{ True } & \multicolumn{2}{|c|}{ False } & \multirow[b]{2}{*}{$\mathrm{N}$} \\
\hline & Hits & $\begin{array}{c}\text { False } \\
\text { Alarms }\end{array}$ & Hits & $\begin{array}{c}\text { False } \\
\text { Alarms }\end{array}$ & \\
\hline $\begin{array}{l}1 \\
2\end{array}$ & $\begin{array}{l}.833 \\
.962\end{array}$ & $\begin{array}{l}.071 \\
.025\end{array}$ & $\begin{array}{l}.860 \\
.964\end{array}$ & $\begin{array}{l}.046 \\
.004\end{array}$ & $\begin{array}{l}10 \\
20\end{array}$ \\
\hline
\end{tabular}

a second study list, B. The false statements that were paired with the List $A$ true statements went into List $B$, and the false statements paired with List B true statements went into List A. ${ }^{1}$ In this way, a pair of lists covering the same range of content areas was constructed.

The 96 sentences constructed in this fashion for each list were preceded by a set of 10 buffer sentences, half true, half false, and followed by a set of 10 sentences, also half true, half false. The function of the buffer sentences was to allow for practice effects to level off and to separate the study sentences from the test. The entire set of 116 sentences was typed on cards. The 20 buffer sentences were kept in fixed order at the beginning and end of the set, but the 96 main sentences were presented in a different random order to each subject.

A single test was constructed by selecting half the true and half the false sentences from each of the two study lists. This meant that a particular test sentence was either new or old for a subject, depending on the study list that he saw. The result of this arrangement was that each subject had in his test list 24 sentences that fell into each of these categories: true new, true old, false new, and false old. These sentences were listed in random order on test sheets that had space next to each sentence for the subject to check "new" or "old."

Procedure. The subject went through the deck of study cards assigned to him at his own pace. In Experiment 1, he simply read the sentences silently; in Experiment 2, he read them aloud and had to say whether each sentence was true or false. In both, he was informed that he would be given a recognition test later but was asked not to try to memorize any item. After the study set was completed, the subject was given a test list consisting of the 96 test sentences and was asked to check each one indicating whether or not it had been on his study set.

Subjects. In Experiment 1, the subjects were 10 undergraduate and graduate students; in Experiment 2, there were 20 undergraduates. In both, half were assigned at random to study List $\mathrm{A}$, the remainder to study List $\mathrm{B}$.

\section{Results}

The mean proportion of hits and false alarms for both studies is shown in Table 2. There was no advantage for the true statements. Whatever difference appeared was in favor of the false statements. The differences were, however, very small and, except for the false alarms in Experiment 2, not significant. The arcsin conversion was used on the proportions before analyzing them.

The results show a slight, anomalous advantage in the memory for the false sentences. It was decided, therefore, to check on the role of two factors: the amount of time subjects spent on true and false sentences, and the effect of distractor sentences.

In both experiments, the subjects controlled the amount of time they spent on each sentence during both study and test. It was possible that subjects were doing 
well on the false sentences at the cost of additional time. To check on this possibility, it was decided to measure the time the subjects took on each sentence during both study and test.

In the two preceding experiments, moreover, it was possible for the subject to respond on the basis of parts of sentences. For example, the word "dog" appeared as the subject of only one sentence in List A. The subject could make the appropriate response "old" to a test sentence containing that word without responding to the entire sentence, whether it was true or false. Similarly, the word "China" did not appear in any sentence in study List $A$. The subject could make the appropriate response "new" to a test sentence containing that word without responding to the entire sentence. It was decided, therefore, to restructure the study lists so that the subject could not respond correctly unless he remembered the entire sentence, both subject and predicate.

\section{EXPERIMENT 3}

Subjects viewed both the study sentences and the test sentences on a computer-controlled display. During the presentation of the study list, they indicated whether each sentence was true or false by pressing one of two buttons. During the presentation of the test, they indicated whether each sentence was new or old, again, by pressing one of two buttons.

\section{Method}

Materials. The basic arrangement for the assignment of new and old sentences was similar to that in Experiments 1 and 2 . There were two study lists. Included in each list were 96 sentences, half true and half false. These 96 sentences were matched pairs as before, with one member in List $A$, the other in List $B$. Half of the true and half the false from each of these sets in Lists $A$ and $B$ appeared in the single test list. ${ }^{2}$ For any subject, then, half the sentences on the test list were new and half old. Each study list included, however, 96 additional sentences, 2 sentences in A matched to each of the 48 items from B that appeared in the test list, and 2 sentences in B that matched each of the 48 sentences from $A$ that appeared in the test list. These matched sentences furnished distractors that made it impossible for the subject to respond accurately if he responded solely on the basis of the subject or predicate of a test sentence. Some examples of study list and test list items are given in Table 3. The 192 study items were preceded and followed by blocks of 10 buffer sentences.

The subject given study List A should, on the test list, answer "old" to "Plato was a painter" and "new" to "The Andes are a mountain range." The subject given study List B should give the reverse responses. The structure of the study lists prevented the subject from responding correctly to the test sentences on the basis of the sentence subjects or predicates alone. The subject had seen, in his study list, all the subjects and predicates that appeared in the new sentences of the test list. If the subject given List A responded "old" on the basis of the familiarity of the phrase "A mountain range," he made an error.

Procedure. The procedure was basically the same as in Experiment 2 except for the following. The successive sentences were viewed on a computer display. The subject did not read the sentences aloud but indicated his responses by keypresses.

The subject sat before the display with the preferred finger of each hand on one of two keys: one labeled "true," the other "false." He first saw the 212 sentences in his study set one at a time and indicated by pressing one of the keys whether he thought the sentence "true" or "false." As soon as the subject responded to a sentence, it was removed and the next sentence appeared. After the completion of this study list, the experimenter labeled the response keys "old" and "new." The test sentences were presented one at a time, and the subject responded to each one. There was no feedback given to the subject during either the study or the test phase. There were four possible assignments of the response keys: "true" and then "old" assigned to the right key, "true" and then "new," "false" and then "old," and "false" and then "new." One-fourth of the subjects was assigned at random to each of those conditions. Within each of these conditions, half the subjects received List A, half List B.

Subjects. The subjects were 24 adults between the ages of 18 and 25 years who were paid for their participation in the experiment. All were right-handed and all were native speakers of English.

\section{Results}

Table 4 displays the data on the subjects' classifications of the true and false sentences during the study phase. There was no overall difference between the two groups with respect to correctness of response. Analysis of variance of the proportions of correct response, transformed as arcsins, found no difference between Groups $A$ and $B(F<1)$. There was a slight but statistically significant tendency to respond more accurately to the false statements $[F(1,22)=19.90, p<.001]$.

The latencies showed no differences between the groups. Analysis of variance of the latencies found neither the effect of groups nor truth vs. falsity significant (for both, $F<1$ ). Separate analyses for the correctly and incorrectly classified statements gave the same results. The absence of a difference in latency in responding to true as opposed to false statements is important because it simplifies the interpretation of any subsequent differences in latency found between the sentence types.

Table 3

Sample Sentences Demonstrating the Structure of the Study Lists and the Test List in Experiment 3

Study List A

Plato was a painter.

Study List B

\section{Plato was an American.}

Roosevelt was a painter.
Plato was a painter.

The Andes are high.

The Rockies are a mountain range. 
Table 4

Proportion of Correct and Incorrect Classifications of Sentences and Their Latencies (in Milliseconds) During the Study Phase of Experiment 3

\begin{tabular}{|c|c|c|c|c|c|c|c|c|}
\hline & \multicolumn{4}{|c|}{ Proportion } & \multicolumn{4}{|c|}{ Mean Latency } \\
\hline & \multicolumn{2}{|c|}{ True } & \multicolumn{2}{|c|}{ False } & \multicolumn{2}{|c|}{ True } & \multicolumn{2}{|c|}{ False } \\
\hline & Correct & Incorrect & Correct & Incorrect & Correct & Incorrect & Correct & Incorrect \\
\hline $\begin{array}{l}\text { List A } \\
\text { List B }\end{array}$ & $\begin{array}{l}.940 \\
.926\end{array}$ & $\begin{array}{l}.060 \\
.074\end{array}$ & $\begin{array}{l}.956 \\
.968\end{array}$ & $\begin{array}{l}.044 \\
.032\end{array}$ & $\begin{array}{l}1612.75 \\
1589.42\end{array}$ & $\begin{array}{l}2106.75 \\
2681.92\end{array}$ & $\begin{array}{l}1618.08 \\
1648.75\end{array}$ & $\begin{array}{l}2046.67 \\
1832.83\end{array}$ \\
\hline Mean & .933 & .067 & .962 & .038 & 1601.08 & 2394.34 & 1638.42 & 1939.75 \\
\hline
\end{tabular}

Table 5

Proportion of Correct and Incorrect Recognition Responses and Their Latencies (in Milliseconds) in the Test Phase of Experiment 3

\begin{tabular}{|c|c|c|c|c|c|c|c|c|}
\hline & \multicolumn{4}{|c|}{ Proportion } & \multicolumn{4}{|c|}{ Mean Latency } \\
\hline & \multicolumn{2}{|c|}{ True } & \multicolumn{2}{|c|}{ False } & \multicolumn{2}{|c|}{ True } & \multicolumn{2}{|c|}{ False } \\
\hline & Correct & Incorrect & Correct & Incorrect & Correct & Incorrect & Correct & Incorrect \\
\hline \multicolumn{9}{|c|}{ New Sentences } \\
\hline $\begin{array}{l}\text { List A } \\
\text { List B }\end{array}$ & $\begin{array}{l}.736 \\
.646\end{array}$ & $\begin{array}{l}.264^{*} \\
.354\end{array}$ & $\begin{array}{l}.622 \\
.670\end{array}$ & $\begin{array}{l}.378 \\
.330\end{array}$ & $\begin{array}{l}2031.66 \\
2564.08\end{array}$ & $\begin{array}{l}1610.36 \\
1948.75\end{array}$ & $\begin{array}{l}2065.83 \\
2557.50\end{array}$ & $\begin{array}{l}2033.67 \\
2905.83\end{array}$ \\
\hline Mean & .691 & .309 & .646 & .354 & 2297.87 & 1779.56 & 2311.66 & 2469.75 \\
\hline \multicolumn{9}{|c|}{ Old (Conditionalized) Sentences } \\
\hline $\begin{array}{l}\text { List A } \\
\text { List B }\end{array}$ & $\begin{array}{l}.873 \\
.897\end{array}$ & $\begin{array}{l}.127 \\
.103^{*}\end{array}$ & $\begin{array}{l}.846 \\
.774\end{array}$ & $\begin{array}{l}.154 \\
.226\end{array}$ & $\begin{array}{l}1559.83 \\
1743.17\end{array}$ & $\begin{array}{l}2404.42 \\
2270.36\end{array}$ & $\begin{array}{l}1763.75 \\
2101.08\end{array}$ & $\begin{array}{l}2089.58 \\
2775.08\end{array}$ \\
\hline Mean & .885 & .115 & .810 & .190 & 1651.50 & 2337.39 & 1932.42 & 2432.33 \\
\hline
\end{tabular}

Note-For new sentences, incorrect recognition responses = false alarms; for old sentences, correct recognition responses $=$ hits. *Based on 11 subjects, since one subject did not make any incorrect responses for this category.

Table 5 shows the mean proportion of correct rejections and incorrect acceptances (false alarms) for the new sentences presented in the test list. $^{3}$ It also shows the mean latencies for each category. Overall, the accuracy of the subjects was slightly higher on the true sentences, but only List A showed this advantage. Analysis of variance of the proportions correct, transformed as arcsins, found no significant effect [groups, $\mathrm{F}(1,22)=1.27, \mathrm{p}>.10$; sentence class, $\mathrm{F}<1$; interaction $F(1,22)=1.94, p>.10]$.

The mean latencies showed no obvious differences between true and false in the correct rejections but a considerable difference in the false alarms. Analysis of variance bore out those findings. For the correct rejections, there were no significant effects $(F<1$, for sentence class). For the false alarms, there was a significant effect for sentence class $[F(1,21)=15.19$, $p<.001]$. The reduction in degrees of freedom reflected the absence of data for one subject who did not make any false alarms. These data also showed a significant difference between the two groups $[F(1,21)=4.62, p<.05]$, but no interaction between groups and sentence class $[F(1,21)=2.68, p>.10]$. The longer latencies for the group that had List $B$ is found in almost all the data in Table 5 .

The analysis of the responses to the old sentences can be carried out either on all the responses to old sentences or only on the sentences the subject classified correctly during the study phase (conditionalized on correct original classification). Only the conditionalized data are presented in Table 5, since the two sets of data are almost identical.

Analysis of variance of the transformed proportions correct for the hits in the conditionalized data found a significant effect of sentence class $[F(1,22)=9.95$, $p<.005]$. The absence of a difference in the false alarm rates reported above makes the interpretation of this difference simple. Neither the effect of groups $(F<1)$ nor the Groups by Sentence Class interaction $[F(1,22)=4.22, p>.10]$ was statistically significant.

Analysis of the latency data for the correct response to the old sentences (hits) in the conditionalized data found a significant effect for sentence class $[F(1,22)=31.38$, $p<.001]$. Neither groups $[F(1,22)=2.38]$ nor the interaction $[F(1,22)=2.36]$ was significant $(p>.10)$. A parallel analysis of the latencies for the misses found no significant effects there [sentence class, $F<1$; groups, $F<1$; interaction, $F(1,21)=1.64, p>.30]$. (Again, the reduction of degrees of freedom reflects the absence of data for one subject who did not miss any old sentences.)

Analysis of variance of the unconditionalized data for the old sentences gave essentially the same picture as the analysis of the conditionalized. The only differences were in the sizes of the Fs and the significance levels.

The difference in the accuracy of the recognition for 
true and false sentences may also be summarized compactly in terms of $d^{\prime}$. The overall $d^{\prime}$ 's based on the mean proportions of hits and false alarms in Table 5 were 1.68 for the true sentences and 1.21 for the false sentences. The d's computed separately for Groups A and B were as follows: for Group A, true-1.78, false-1.27; for Group B, true-1.65, false-1.14.

Analyses were also carried out comparing the latencies for the correct rejections with the misses and comparing latencies for the hits with the false alarms within both the true and the false sentences. The results showed that there was no significant difference between the latencies for the correct rejections and the misses in either sentence class [for true, $F<1$; for false, $F(1,22)=2.71, p>.10]$. Comparison of the latency for the hits with the latency for the false alarms found the following effects [for true sentences, $F(1,21)=3.61$. $p<.10$; for false sentences, $F(1,22)=12.57, p<.005$ ]

The analyses above involved 10 comparisons over Table 5. To guard against the effects of an increase in experiment error rate, the Bonferroni t (Myers, 1972) was used, correcting the significance levels for those comparisons. With that statistic, the interpretation of the results described above remained essentially the same for the critical comparisons of true vs. false statements, as well as the comparisons of hits vs. false alarms within both the true and the false. Those effects significant at the .001 level were then significant at the .01 level. Those significant at the .005 level were then significant at the .05 level. The only effect that dropped out was the difference between the hits and the false alarms for the true statements, which was originally borderline at $\mathrm{p}<.10$.

The results with respect to latencies may be summarized as follows. For all cases in which the subject responded "new" (i.e., for both correct rejections and misses and for both true and false), the latencies were about the same. Differences appeared when the subject responded "old." Hits for true statements took less time than hits for false statements. False alarms showed the same pattern. Within true sentences as well as within false sentences, the hits took less time than the false alarms.

\section{DISCUSSION}

The data of the last experiment demonstrate clear superiority of recognition memory for true as opposed to false sentences. The findings fit the hypothesis of double representation of true statements in a memory task. This hypothesis is also supported by the two facts cited earlier: (1) Encoding tasks that call on the subject's basic knowledge have greater effect than tasks that do not. (2) Among those encoding tasks, the ones that generate a positive response have greater effect than those that do not.

The latency data in general support the hypothesis.
Hits for true sentences have significantly lower latencies than hits for false sentences, as might be expected if true old sentences furnish two sets of information leading to the response "old." False alarms for true sentences take less time than false alarms for false sentences, as might be expected if both classes can be incorrectly marked in episodic memory while only the true sentences can be marked, incorrectly here, in the store of knowledge. All "new" responses are approximately $2,300 \mathrm{msec}$, both correct rejections and misses for both true and false sentences. This finding suggests that the subjects, when faced with a sentence that appears not to be marked, continue their search of memory until a time limit expires and then respond "new."

The reason for the differences between the results of Experiment 3, which did find differences in recognition memory for true and false sentences, and the results of Experiments 1 and 2, which did not, probably lies in the type of distractor used in Experiment 3. The subject could not, in Experiment 3, make a correct response on the basis of familiarity of part of a sentence. This explanation implies two other propositions. One is that the subject faced with a sentence stores information about both the sentence and parts of the sentence that he can use in a later recognition test. The other is that the subject can choose which of his sets of data he makes use of during the recognition test. If he perceives that sentence parts give him unambiguous information, he uses the sentence parts. If he perceives that they do not, then he uses only the complete sentences. This means that the subject is sensitive to the clarity of available information sources without special instruction or feedback. No such feedback was presented in these experiments.

\section{REFERENCES}

BADDELEY, A. D. The trouble with levels: A reexamination of Craik and Lockhart's framework for memory research. Psychological Review, 1978, 85, 139-152.

Cratk, F. I. M., \& Lockhart, R. S. Levels of processing: A framework for memory research. Journal of Verbal Learning and Verbal Behavior, 1972, 11, 671-684.

Craik, F. I. M., \& Tulving, E. Depth of processing and the retention of words in episodic memory. Journal of Experimental Psychology: General, 1975, 104, 268-294.

Glanzer, M., \& Koppenaal, L. The effect of encoding tasks on free recall: Stages and levels. Joumal of Verbal Leaming and Verbal Behavior, 1977, 16, 21-28.

Moscovitch, M., \& Craik, F. I. M. Depth of processing, retrieval cues, and uniqueness of encoding as factors in recall. Journal of Verbal Learning and Verbal Behavior, 1976, 15, 447-458.

Myers, J. L. Fundamentals of experimental design (2nd ed.). Boston: Allyn \& Bacon, 1972.

Scarborough, D. L., Cortese, C., \& Scarborough, H. S. Frequency and repetition effects in lexical memory. Journal of Experimental Psychology: Human Perception and Performance, 1977. 3. 1-17.

Schulman. A. I. Recognition memory for targets from a scanned word list. British Joumal of Psychology, 1971, 62, 335-346. 
Schulman, A. I. Memory for words recently classified. Memory \& Cognition, 1974, 2, 47-52.

Tulving, E. Episodic and semantic memory. In E. Tulving \& W. Donaldson (Eds), Organization of memory. New York: Academic Press, 1972.

\section{NOTES}

1. The paired true and false sentences were generated in the following way. A triad of sentences of the same form on the same substantive area was constructed (e.g., "A hoop is used in basketball," "A puck is used in hockey," "A bow is used in archery"). At random, one of the three was chosen as a true sentence (as in the example above, that sentence was "A puck is used in hockey"). Again at random, the subject of one of the remaining two was chosen to be combined with the predicate of the other remaining sentence to form the paired false sentence (in the example above, "A bow" plus "is used in basketball").

2. Through a programming error, however, only 23 of the 24 old sentences were included in the test set for List A.

3. False alarm rates are higher in Experiment 3 than in Experiment 1 or 2 , as would be expected on the basis of the structure of the distractors.

(Received for publication August 3, 1978; revision accepted December 12, 1978.) 